\title{
Is Magnetic Resonance Imaging Superior to Conventional Radiography in Neonates with Anorectal Malformation?
}

\author{
Sarita Chowdhary ${ }^{1}$ Dinesh Kumar ${ }^{2}$ Shyamendra Pratap Sharma ${ }^{1} \quad$ S. P. Sharma ${ }^{1}$ Pranay Panigrahi ${ }^{1}$ \\ ${ }^{1}$ Department of Paediatric Surgery, Institute of Medical Sciences, \\ Banaras Hindu University, Varanasi, Uttar Pradesh, India \\ 2Department of General Surgery, Motilal Nehru Medical College, \\ Allahabad, Uttar Pradesh, India

\begin{abstract}
Address for correspondence Sarita Chowdhary, MBBS, MS, MCh, Department of Paediatric Surgery, Institute of Medical Sciences, Banaras Hindu University, Varanasi 221005, Uttar Pradesh, India (e-mail: saritaimsbhu@yahoo.com).
\end{abstract}

Ann Natl Acad Med Sci (India) 2020;56:38-41

\begin{abstract}
Introduction This study was conducted to evaluate the role of magnetic resonance imaging (MRI) in neonates with anorectal malformation (ARM).

Materials and Methods Newborns, referred to Pediatric Surgical Ward through General Surgery Outpatient Department and Emergency Department of Sir Sunderlal Hospital as a case of ARM, were included in the study. Unstable patients requiring urgent primary colostomy were excluded from the study. Forty neonates with ARM and 20 neonates having no ARM were included in the study ( 2 years, i.e., conducted between September 2018 and July 2020). The included patients were evaluated clinically and were subjected to an invertogram (after 24 hours of delivery), which is conventional radiography. Further, MRI was performed using $1.5 \mathrm{~T}$ superconductive system; 5 to $6 \mathrm{~mm}$ contiguous sections were obtained using a head or a body coil according to the size of the infant patient to obtain images with sharp anatomical detail: Sagittal, coronal, and axial conventional spin-echo T1-weighted images of the pelvic region were obtained in all patients, with perpendicular coronal plane and the axial plane parallel to pelvic floor.

Results The majority of patients (60\%) were referred on day 3 of birth and rest were referred after 3 days of birth. Conventional radiography, that is, invertogram was done in all study population. Sixty per cent of the total included patients had supralevator location of rectal pouch. Conventional radiography was detected to be low type of

Keywords

- anorectal malformation

- invertogram

- MRI

- sphincter muscle complex

- external anal sphincter

- VACTERL ARM-all had infralevator position of rectal pouch in MRI, but $60 \%$ of the intermediate had supralevator level and all high abnormalities had supralevator level. These distribution anomalies were statistically significant. The levator ani length index and external anal sphincter length index were also compared and found to be thickest in intermediate type, thick in low types, and thin in high type of abnormalities, which is an added advantage of MRI over radiography.

Conclusion Preoperative assessment of ARM with MRI gives useful information regarding type of malformation as well as sphincter muscle and associated malformations. Large sample size was needed for future landmark in the field of ARM.
\end{abstract}

DOI https://doi.org/

10.1055/s-0040-1710163

ISSN 0379-038X. (c)2020 National Academy of Medical Sciences (India)
License terms

()(1) $\Theta \circledast$ 


\section{Introduction}

Anorectal malformations (ARMs) are relatively rare congenital anomalies, with a reported incidence of around 3:10,000 live births. ${ }^{1}$ Temporarily, ARMs are treated with colostomy. Usually, reconstruction is planned as early as possible to achieve good bowel control. ${ }^{2}$ Exact type of ARM and fistula is needed for definite repair. Although several classification systems for ARMs have been proposed, the Krickenbeck classification-based on the presence/absence of a fistula and its location-is the most widely accepted., ${ }^{2,3}$ The posterior sagittal anorectoplasty (PSARP), as described by Peña and Devries is commonly used surgical technique for repair of ARMs; however, this technique can be used when fistula ends below or at the level of the pelvic floor (levator ani muscle). ${ }^{4}$ Higher positioned fistulas, mainly the rectovesical or prostatic rectourethral need a laparoscopic or laparotomic approach. ${ }^{2}$ In cases of longer fistulas ending below the pelvic floor but with a distal rectum ending higher above the pelvic floor, the PSARP may not be sufficient to complete the reconstruction, and laparoscopy or laparotomy may also be required. Preoperative imaging is thus mandatory for the surgeon to decide the surgery.

\section{Materials and Methods}

\section{Study Setting and Patients Selection}

It is an observational study and conducted between September 2018 and July 2020 in the Department of Paediatric Surgery. Approval was obtained from the Institutional Ethics Committee of Institute of Medical Sciences, Banaras Hindu University. Newborns, referred to Pediatric Surgical Ward through General Surgical Outpatient Department and Emergency Department of Sir Sunderlal Hospital, were admitted as a case of ARM. Newborns of less than 1 month of age were included in the study. Unstable patients requiring urgent primary colostomy were excluded from the study.

\section{Patient Evaluation and Procedure}

The included patients were evaluated clinically and subjected to invertogram (after 24 hours of delivery), which is conventional radiography. MRI (1.5 T Siemens MAGNETOM Avanto workstation [software Numaris/4, Version Syngo MRB17]) was performed using $1.5 \mathrm{~T}$ superconductive system; 5 to $6 \mathrm{~mm}$ contiguous sections were obtained using a head or a body coil according to the size of the patient to obtain a sharp image with sharp anatomical detail. T1-weighted images with a spin-echo (SE) pulse sequence and a short repetition time (400-600 milliseconds) and echo time (20 milliseconds) were obtained in all patients. T2-weighted images were obtained in selected patients. T1-weighted images are better for morphological description, which is the area of interest in the study.

Sagittal, coronal, and axial conventional SE T1-weighted images of the pelvic region were obtained in all patients. Coronal plane perpendicular and the axial plane parallel to the pelvic floor were taken. T2-weighted images in different planes were obtained, when associated anomalies of the spinal cord, spine, or kidneys were detected. A head coil was used in infants. The slice thickness was kept as thin as possible (3-5 mm) with a small interslice gap (usually $1.25 \mathrm{~mm}$ ).

MRI scan was done for levator ani muscle and external anal sphincter (EAS) thickness. Interischial distance was measured at direct coronal section (i.e., maximum thickness). Evaluation was done at the level of rectal pouch in relation to levator ani muscle. MRI was also evaluated for any vertebral column, renal abnormalities. Control patients were taken up for muscle index comparisons from nongenital anomalies of same age group.

\section{Statistical Analysis}

Statistical analysis was done using SPSS 16 , Student's $t$-test. One-way analysis of variance was used to compare the significant difference of mean. For categorical variables, chi-square test and Fisher's exact test were used. A p-value of $<0.05$ was considered as statistically significant.

\section{Results}

The majority of patients (60\%) presented at third day of birth. Eighty per cent of patients were male. Maximum number of patients belonged to weight 2.1 to $3.0 \mathrm{~kg}$. Sixty per cent of the patients had supralevator rectal pouch. Sphincter muscle complex (SMC) was visualized in the MRI films and was found to be well developed in $40 \%$ of the cases. All the patients with low type of deformity had well-developed SMC complex. All patients with intermediate type of deformity had good SMC complex and 66\% of the patients with high deformity had good SMC development in MRI scans. This distribution was statistically significant.

\section{Spinal Abnormality on MRI}

Sixty per cent of the patients in the study were normal spine and the other had some kind of spinal deformity. It revealed normal lower spine in all of the patients with low type of abnormality, whereas $80 \%$ patients with high type of deformity revealed some kind of spinal abnormality.

\section{Urological Abnormality on MRI}

Forty per cent of the intermediate types had normal urological tract and $66.7 \%$ of the high abnormalities had normal urological tract. The data were statistically significant.

\section{Muscle Development}

The mean of the thickness of the levator ani and EAS in each group was evaluated and the difference was found to be statistically significant in levator ani thickness. The difference in interischial distance, the marker of pelvic size, was also significant between diagnoses diminishing the role of muscle thickness comparison between diagnoses. The levator ani length index and EAS length index were also compared and they were thickest in the intermediate type, thick in low types, and thin in high type of abnormality. These differences were statistically significant. Levator ani length index and EAS length index were correlated significantly with striated muscle complex development. The association was highly 
significant. The difference in muscle length index of levator ani between patients with infralevator pouch position and supralevator pouch position was statistically significant.

Out of 40 cases, 16 patients had infralevator rectal pouch and 24 had supralevator level of rectal pouch. SMC was well developed in $50 \%$ of infralevator and $33.3 \%$ of supralevator rectal pouch cases. The distribution of the SMC development compared with the level of rectal pouch was not statistically significant.

\section{Discussion}

ARM is a spectrum of congenital disorders affecting the distal gut primarily anorectal region and lower urogenital system. Varied surgical intervention, ranging from anoplasty to complex surgical procedures can be done. Thus, varied outcomes come from good to poor bowel control. ${ }^{5}$ The entity of ARM has been in the literature since ancient Greek times with Hippocrates to Aristotle mentioning and attempting to treat it. ${ }^{6}$

As compared with our previous article, which was on postoperative MRI evaluation of ARM patients with clinical correlation in 2013, this time, the study was on preoperative evaluation with higher version of MRI machine. This study was novel in the way that it included neonates $(n=40)$ for the preoperative work-up, whereas McHugh et al in 1995 included infants $(n=9)$, and Taccone et al included infants and toddlers $(n=5)$. Accurate evaluation of children with ARM involves correct assessment of the level, type of malformation, the existence of fistula, the developmental state of the SMC, and the presence of associated anomalies. ${ }^{7,8}$ This information is essential in planning initial management, as well as predicting morbidity, quality of life, and prognosis of survival. Since its introduction, MRI has been proven to be best modality to answer these crucial questions, which is supported by the results of the current study.

\section{Associated Anomalies}

VACTERL anomalies include anorectal, cardiac, esophageal, renal, and limb anomalies. Surgical result and prognosis of neonate depend on associated anomalies. ${ }^{9}$ Urological anomalies occur in 28 to $72 \%$ of cases. ${ }^{10}$ Sixty per cent of the patients were also found to have some kind of urological abnormality such as absent kidney, hydroureteronephrosis, etc. Urological abnormalities were found to be 37,33 , and $35 \%$ in the studies by Nievelstein et al, McHugh et al, and Taccone et al, respectively. Thomeer et al, on the other hand, found comparable results to this present study, that is, $60 \%$ of the patients with urological abnormality. ${ }^{11}$

This study also found that $60 \%$ of the patients had some kind of a spinal abnormality from hemivertebra to spinal dysraphism too. Previous studies depicted 33 and 25\% abnormality by McHugh et al and Taccone et al, respectively, whereas Nievelstein et al and Thomeer et al reported comparable outcomes in their study where the incidence of spinal abnormalities were 51 and $55 \%$, respectively.
Some studies in the literatures show some spinal anomalies such as myelodysplasia despite having normal radiography. ${ }^{11}$ So, neonates with normal ultrasonography of the spine and radiography should undergo MRI spine to rule out spinal anomalies with ARM patients. ${ }^{12-14}$

\section{Practical Anatomy}

Levator ani muscles, puborectalis muscle, and EAS (have superficial, subcutaneous, deep) are important for continence. ${ }^{15}$ Axial MRI through puborectalis and EAS is important for muscle assessment. ${ }^{16}$

Pubococcygeal (PC) line and ischial line are important for classification of ARM. Rectal pouch above ischial and PC lines is high-type ARM, between PC and ischial lines is intermediate, and low-type ARM when rectal gas is below both lines. ${ }^{14}$ The puborectal and EAS muscles can be subjectively assessed on all imaging planes in terms of size as good, mediocre, or poor. Some studies have used actual measurements of the thickness of the sphincteric muscles as a guide to their overall development. Subjective visual evaluation of the sphincteric muscles without strict measurements is generally adequate. ${ }^{17}$

EAS and levator ani muscle, its development and its association were thoroughly studied in this study. The study found that the levator ani length index and EAS length index were thickest in intermediate type, thick in low types, and thin in high type of abnormality. These differences were statistically significant. The deficiency in levator ani and EAS muscle length index was reflected in the amount of abnormality in urological tract in MRI scans. The levator ani length index and EAS length index were correlated in statistically significant manner with the normal spinal evaluations on MRI scan. Levator ani length index and EAS length index were correlated significantly with SMC development.

PSARP surgery utilizes puborectalis and EAS muscles for it. 7.16 Previously, these muscles were not used for continence. Nowadays, it is very important for prognosis. MRI gives valuable information regarding thickness, normally placed or not. ${ }^{16}$

\section{Newborn Period}

Newborn needs to classify into high, intermediate, and low types for decision of surgery. A prone cross-table lateral plain radiograph or "invertogram" was performed to look for rectal pouch location. In the 10 to $20 \%$ of neonates with equivocal clinical findings, radiology can have significant inherent drawbacks. Decisions to be made during this period are whether the patient needs a colostomy or whether other urinary or vaginal diversions to prevent likely sepsis or acidosis are necessary. ${ }^{18}$ Straining or crying can cause the puborectal muscle to move 2 to $3 \mathrm{~cm}$ in the same patient. ${ }^{19}$ Examination during sedation or sleep may give a more accurate estimation of the true level of the levator sling. ${ }^{16}$ Patients who were detected to be low type of ARM in radiography had infralevator position of rectal pouch in MRI. Sixty per cent of the intermediate had supralevator level and all high abnormalities had supralevator level in MRI. These distributions were statistically significant. 


\section{Limitation of the Study}

Large sample size is needed for future landmark in the field of ARM.

\section{Conclusion}

A better understanding of the pelvic anatomy is needed for future ARM management and its prognostication. Keeping the trend of primary neonatal definitive repairs for better functional outcomes and lesser morbidity, this study was aimed to access the viability of MRI as an important preoperative imaging modality as compare with conventional radiology (invertogram).

MRI provides a detailed anatomy of the pelvic musculature and SMC in neonatal pelvis which provides a route map to surgery. MRI detected lower vertebral and urological anomalies. The dictum that the higher the deformity, the more the chances of spinal deformities was supported, whereas the level of defect did not correlate in the same way for urological abnormalities. MRI is armed with detailed visualization and radiation-free imaging but takes a long time and requires sedation.

\section{Authors' Contributions}

S.C. compiled all data and wrote the article. D.K. recorded data and followed-up with patients. P.P. and S.S. did statistical analysis of data. S.P. overall monitored the article. All authors went through the article and permit it with full agreement.

\section{Note}

This study was conducted with the help of Radiology Department and Paediatric Surgery Department of Banaras Hindu University, Varanasi.

\section{Ethical Approval}

This study was approved by Research Ethical Committee of Faculty of Medicine at BHU Committee room on February 18, 2014, with reference no. Dean /2014-15/ $\mathrm{EC} / 412$. Informed written consent was taken from parents of neonates. Hard copy of consent is present with authors.

\section{Funding}

None.

\section{Conflict of Interest}

None declared.

\section{References}

1 Wijers CH, van Rooij IA, Marcelis CL, Brunner HG, de Blaauw I, Roeleveld N. Genetic and nongenetic etiology of nonsyndromic anorectal malformations: a systematic review. Birth Defects Res C Embryo Today 2014;102(4):382-400
2 Alamo L, Meyrat BJ, Meuwly JY, Meuli RA, Gudinchet F. Anorectal malformations: finding the pathway out of the labyrinth. Radiographics 2013;33(2):491-512

3 Holschneider A, Hutson J, Peña A, et al. Preliminary report on the International Conference for the Development of Standards for the Treatment of Anorectal Malformations. J Pediatr Surg 2005;40(10):1521-1526

4 Peña A, Devries PA. Posterior sagittal anorectoplasty: important technical considerations and new applications. J Pediatr Surg 1982;17(6):796-811

5 Levitt MA, Peña A. Anorectal malformations. Orphanet J Rare Dis; $2007 ; 2: 33$

6 Tsoucalas G, Gentimi F, Kousoulis AA, Karamanou M, Androutsos G. Imperforate anus and perianal fistula in ancient Greek medical writings. Ann Gastroenterol 2012;25(3):241-242

7 McHugh K, Dudley NE, Tam P. Pre-operative MRI of anorectal anomalies in the newborn period. Pediatr Radiol 1995;25(Suppl 1) :S33-S36

8 Nievelstein RA, Vos A, Valk J, Vermeij-Keers C. Magnetic resonance imaging in children with anorectal malformations: embryologic implications. J Pediatr Surg 2002;37(8):1138-1145

9 Smith ED. The bath water needs changing, but don't throw out the baby: an overview of anorectal anomalies. J Pediatr Surg 1987;22(4):335-348

10 Thomeer MG, Devos A, Lequin M, et al. High resolution MRI for preoperative work-up of neonates with an anorectal malformation: a direct comparison with distal pressure colostography/fistulography. Eur Radiol 2015;25(12):3472-3479

11 Taccone A, Martucciello G, Dodero P, et al. New concepts in preoperative imaging of anorectal malformation. New concepts in imaging of ARM. Pediatr Radiol 1992;22(3):196-199

12 Long FR, Hunter JV, Mahboubi S. Kalmus A, Templeton JM Jr. Tethered cord and associated vertebral anomalies in children and infants with imperforate anus: evaluation with MR imaging and plain radiography. Radiology 1996;200(2):377-382

13 Beek FJ, Boemers TM, Beek FJ, et al. Spine evaluation in children with anorectal malformations. Pediatr Radiol 1995;25(Suppl1): S28-S32

14 Heij HA, Nievelstein RA, de Zwart I, Verbeeten BW, Valk J, Vos A. Abnormal anatomy of the lumbosacral region imaged by magnetic resonance in children with anorectal malformations. Arch Dis Child 1996;74(5):441-444

15 deVries PA, Cox KL. Surgery of anorectal anomalies. Surg Clin North Am 1985;65(5):1139-1169

16 Sato Y, Pringle KC, Bergman RA, et al. Congenital anorectal anomalies: MR imaging. Radiology 1988;168(1):157-162

17 Fukuya T, Honda H, Kubota M, et al. Postoperative MRI evaluation of anorectal malformations with clinical correlation. Pediatr Radiol 1993;23(8):583-586

18 Peña A. Management of anorectal malformations during the newborn period. World J Surg 1993;17(3):385-392

19 Berdon WE, Baker DH, Santulli TV, Amoury R. The radiologic evaluation of imperforate anus. An approach correlated with current surgical concepts. Radiology 1968;90(3):466-471 